ORIGINAL ARTICLE

\title{
Liquid based cytology: examination of its potential in a chlamydia screening programme
}

\author{
J Hopwood, H Mallinson, E Hodgson, L Hull
}

Sex Transm Infect 2004;80:371-373. doi: 10.1136/sti.2003.008359

See end of article for authors' affiliations

.....................

Correspondence to: Dr J Hopwood, Chlamydia

Office, St Catherine's Hospital, Birkenhead CH42 OLQ, UK;

drihopwood@aol.com

Accepted for publication 7 December 2003

\begin{abstract}
Objective: To assess the feasibility of testing for chlamydia directly on a single liquid based specimen (ThinPrep test) collected for cervical screening.

Method: Cervical smears were taken using a Cervex spatula and rinsed in the liquid based cytology collection vial. Following this, the conventional sample for chlamydia testing was taken from the endocervix using an Abbott Collection kit. Cytological specimens were prepared using an automated slide processor. Residual cellular material and the conventional samples were sent to the laboratory where both were tested for chlamydia by ligase chain reaction (LCR). The manufacturer's protocol for LCR urine testing was modified to substitute $1 \mathrm{ml}$ of PreservCyt suspension.

Results: 581 women had both swab and cytology suspension tested for Chlamydia trachomatis with LCR. There were 19 concordant positive and 562 concordant negative reports. The stability of chlamydia in the cytology suspension was maintained for at least 5 months.

Conclusion: The findings lead us to conclude that samples collected for liquid based cytology using the ThinPrep test collection vial provide a potential platform for chlamydia screening, though the study established several issues to be addressed to make this a practical proposition.
\end{abstract}

T he potential complications associated with undetected chlamydial infection present not only a serious personal health risk but also a significant public health issue, with the cost to the UK health service estimated at $£ 100$ million annually. ${ }^{1}$ The National Strategy on Sexual Health and HIV Services, launched by the UK government, includes the recommendation that all women attending family planning clinics or going for their first cervical smear be offered chlamydia screening.

The Department of Health funded a pilot screening programme (August 19 to September 2000) in Wirral and Portsmouth using a urine ligase chain reaction (LCR) test. Women were offered testing in general practice, family planning clinics and young people's services and in gynaecology, colposcopy, antenatal and genitourinary medicine departments. An overall positivity of $9.5 \%$ was found in those tested. ${ }^{2}$ The community-wide programme has continued on Wirral. Results are sent to all having the test and people testing positive are managed by the programme's team, which includes community health advisers.

We have found in the pilot and previous studies that the time of cervical screening is an acceptable opportunity to offer chlamydia testing and high positive rates are found in women under 25 years old. ${ }^{3}$ To improve the offer and to enable a more informed choice, one option for delivering this dual screening would be to include information about chlamydia to all women at the time of their first invitation for a smear

The advent of liquid based cytology (LBC) provides a platform for the detection and evaluation of cervical cellular abnormalities and concurrent testing for a variety of sexually transmitted infections including chlamydia. The ThinPrep Pap test (Cytyc UK Ltd, Crawley, Sussex.) is an LBC system that was approved for use in the United States by the US Food and Drug Administration (FDA) in 1996. Briefly, with this method, cervical specimens are collected with a sampling device; then, instead of smearing the cells on a slide, the device is rinsed into a vial containing PreservCyt transport medium. The vial is labelled and sent to the laboratory for slide preparation. Since approval, this method has been evaluated in a variety of published clinical studies and has demonstrated improved cytological detection of cervical abnormalities. ${ }^{4-7}$ In addition, the ThinPrep system has also been approved by the US FDA for adjunctive testing for HPV DNA and for chlamydia and gonorrhoea testing using the residual cellular material remaining in the vial after slide preparation. A number of published studies have demonstrated the ability to perform a variety of adjunctive tests from this collection platform. ${ }^{8-11}$

The objective of this study was to assess the feasibility of testing for chlamydia using LCR directly from a single ThinPrep Pap test specimen collected for cervical screening. We wanted to examine the logistics and practicalities involved at all stages, including time involved, cost and contamination issues, and to compare the performance of chlamydia testing using the LBC specimen with the traditional method.

In Wirral's colposcopy service chlamydia testing is routinely offered to all women under 30 years. Chlamydial infection may contribute to the cytological abnormality which may resolve after its treatment; it could be the cause of a clinical referral such as abnormal bleeding or appearance of the cervix; it could result in treatment morbidity

The ThinPrep 2000 Processor was already funded for use in Arrowe Park Hospital, but for non-gynaecological work only. Funding was made available from Wirral's chlamydia screening programme to use LBC for colposcopy generated cervical smears to enable the study to be carried out. Cytyc supported training for laboratory staff in the interpretation of the ThinPrep smears.

\section{METHODS}

\section{Patients and specimen collection}

Departments involved were Arrowe Park Women's Directorate colposcopy service, the genitourinary medicine department colposcopy service, a general practice colposcopy

Abbreviations: LBC, liquid based cytology; LCR, ligase chain reaction 
service, Arrowe Park Histopathology Department, Liverpool Public Health Laboratory (PHL) and the Wirral Chlamydia Office. Information sheets for patients and a protocol were developed and ethical approval obtained. From May 2001 to May 2002, women attending for colpsocopy were given the information and those having a smear as part of diagnosis or follow up were offered the dual testing of cervical smear and chlamydia in addition to a conventional endocervical swab for chlamydia testing.

Cervical smears were taken using a Cervex spatula rotated five times and vigorously rinsed in the bottle containing PreservCyt fluid. Following this, the conventional sample for chlamydia testing was taken from the endocervix using an Abbott LCx swab collection kit.

A linked request for a chlamydia test on the suspension was transmitted to the cytology laboratory by attaching a chlamydia reference number (CRN) from the specially designed microbiology request form (RF) to the standard cervical smear request form $\mathrm{HMR} / 101 / 5$. The bottles with HMR 101 together with the swabs in the microbiology RF bag were checked for correct identification and taken to the cytology laboratory daily.

Throughout the process, standard procedures were in place to prevent cross contamination of all samples. No extra measures were introduced.

\section{Sample preparation and transport}

The LBC specimen vials along with their accompanying swabs in microbiology RF/bag and appropriate forms were taken to the cytology laboratory (Arrowe Park Hospital Cytology Laboratory) for processing. Samples were checked into the laboratory and because the currently used computer program was not designed to manage this, a day book was kept. A record was kept of the order the samples went into the machine to enable monitoring of results following a positive chlamydia test.

Cytological specimens were prepared using an automated slide processor (ThinPrep 2000 System, Cytyc UK Ltd, Crawley, Sussex) according to the manufacturer's specifications. Slides were Pap stained, screened for cellular abnormalities, and reported according to institutional procedures. There are no national guidelines for reading and reporting LBC smears, so for this study senior medical scientists both screened and checked the smears, though for traditional smears they would not carry out the first screen.

The endocervical swabs in microbiology RF bags were placed in the refrigerator.

Only when it was determined that there was satisfactory cellular content in the smear, was the residual material in the PreservCyt fluid in its container placed in a second microbiology RF linked to the one containing the swab.

These samples were sent in existing transport arrangements to Liverpool PHL. There, both swabs and suspension were tested for chlamydia by LCR (LCx Probe System, Abbott Laboratories, Abbott Park, IL, USA). The manufacturer's protocol for urine which utilises a centrifuged deposit was modified to substitute $1 \mathrm{ml}$ of PreservCyt cell suspension as the sample in the machine. Owing to the high methanol content of PreservCyt fluids they were discarded via the histology department's existing procedure for disposal of flammable liquids.

Following the algorithm used in the chlamydia screening pilot, samples reactive by chlamydia LCR were retested by LCR test on the same sample. Polymerase chain reaction (Roche Cobas) was used as arbiter if there were discrepant LCR results. Communication of results followed current practice; chlamydia results were transmitted only to the colposcopy unit; cervical smear reports were sent to the colposcopy unit with copies to general practitioners. Women with positive chlamydia tests were managed either in the colposcopy unit or in the department of genitourinary medicine according to long standing protocols and practice.

\section{RESULTS}

During the 12 month period from May 2001 to May 2002, 581 women had both swab and suspension tested for Chlamydia trachomatis with LCR. In total, there were 19 concordant positive and 562 concordant negative reports.

For the first 2 months, women of all ages were offered the test. There were no positive results in women over 30 years old. This finding is consistent with previous chlamydia prevalence studies and therefore, screening was then restricted to women under 30 years old.

One case was reported negative following a pattern of testing that showed an LCR negative fluid but some initial reactivity of the LCR swab. Two cases were reported negative following a pattern of testing that showed an LCR negative swab but some initial reactivity of the LCR on fluid. Follow up PCR was negative for all three cases. Such patterns were known to occur with the then current batches of LCR kits. ${ }^{12}$ For the two fluids initially reactive but reported negative, processing through the ThinPrep 2000 Processor followed processing of another patient's fluid with a negative chlamydia result. This leads us to assume there was not an issue of cross contamination during the slide preparation stage.

The stability of chlamydia in positive fluids was examined by retesting 16 of the LBC specimens after they had been held at ambient temperature for several months. All of 16 retested up to the time of reporting have remained positive by LCR for at least 5 months.

\section{DISCUSSION}

In this direct comparison of testing for $C$ trachomatis specimens collected using traditional swab with specimens collected in PreservCyt cytology collection medium we found no discrepant cases between the two methods. In addition, given the $100 \%$ concordance and the fact that each of the 19 positive cases was followed by a negative case during the slide- processing phase, we have no evidence of cross contamination using the ThinPrep 2000 processor.

These findings lead us to conclude that samples collected for fluid based cytology using the ThinPrep Pap test collection vial provide a viable platform for chlamydia screening.

Our findings are consistent with previous studies suggesting that chlamydia screening is best applied to women under 30 years of age. As noted, since during the first 2 months of the study there were no positive results in women over 30, we restricted our study to women under 30 .

Even though we restricted our population to younger women, we found a lower chlamydia positivity than we had ever experienced in the colposcopy setting. However, in this study testing took place at all visits, which include assessment, treatment, and follow up, so some women had already attended and been tested and managed if positive. Also, it is likely that the active screening programme throughout Wirral meant that many of those referred had already been screened in general practice. Further examination showed that where we could establish the status of the visit 9/108 (8.3\%) tested positive at their first visit, and 2/175 (1.7\%) tested positive at subsequent visits. We believe this supports the premise that the lower than expected overall prevalence of chlamydial infection in this population is attributable to previous detection and treatment.

One option of an equitable opportunity for women to have a chlamydia test, which would facilitate a community message and inform about other testing options is to send information about chlamydia with the first smear invitation. 
This gives an opportunity for an informed decision about the test. A request for chlamydia testing can be made clearly via the smear request form, in this study by using adhesive chlamydia labels.

If LBC is used, then this study confirms that use of its residual suspension would be possible for chlamydia testing. The LCR modified urine test on PreservCyt performed well. There seems to be excellent stability of chlamydia in the fluid so that transport and storage would not be a restricting factor, but clearly any backlog affecting cytology preparation could potentially lead to an unacceptable delay in testing for chlamydia where timely treatment is all important. This study involved some change of practice in the cytology laboratory to expedite sending of samples to the microbiology laboratory.

In a colposcopy setting in this study no special measures were required for safe storage of these small volumes of flammable liquid but in larger scale programmes this may need to be addressed by both clinics and laboratories.

Results and management of those who screened positive were dealt with by the colposcopy service according to current practice. However, there is not a nationwide system that could support this in a community based programme. The current call-recall mechanism for the NHS cervical screening programme could not manage the recording of dual tests and results. However, in Wirral where there is a well defined support system for chlamydia screening, dual testing could readily be managed. Despite this logistical difficulty, the other advantages that could facilitate delivery of an equitable programme suggest that further work on this is required.

\section{Note}

Since this study there is NICE approval for LBC in the cervical screening programme (www.nice.org.uk). Although it is now recommended that cervical screening should commence at 25 years rather than 20 years, the time of first smear may still represent an opportunity for those who have never accessed or been offered a chlamydia test previously and this could even have the potential to contribute to the measure of uptake and coverage of the chlamydia screening programme (Sasieni P, Adams J, Cuzick J. Benefits of cervical screening at different ages; evidence from the UK audit of screening histories. British Journal of Cancer July 2003, www.cancerscreening. nhs.uk ages; evicen)

\section{ACKNOWLEDGEMENTS}

Thanks to the staff in the colposcopy, cytology, and microbiology departments, and especially to Helen Jeffs.

\section{Authors' affiliations}

J Hopwood, Chlamydia Office, St Catherine's Hospital, Birkenhead, Wirral, UK

H Mallinson, L Hull, Liverpool PHLS Laboratory, University Hospital at Aintree, Lower Lane, Liverpool L9 7AL, UK

E Hodgson, Histopathology Department, Arrowe Park Hospital, Wirral, UK

\section{REFERENCES}

1 Chief Medical Officer's Expert Advisory Group. Main report of the CMO's expert advisory group on Chlamydia trachomatis. London: Department of Health, 1998

2 Department of Health, England. Sexual health and HIV section (website www.doh.gov.uk/sexualhealthandhiv/index.htm).

3 Hopwood J, Mallinson H. Chlamydia testing in community clinics: a focus for accurate sexual health care. Br J Fam Plann 1995;2:87-90.

4 Bernstein SJ, Sanchez-Ramos L, Ndubisi B. Liquid-based cervical cytologic smear study and conventional Papanicolaou smears: a metaanalysis of prospective studies comparing cytologic diagnosis and sample adequacy. Am J Obstet Gynecol 2001;185:308-17.

5 Diaz-Rosario LA, Kabawat SE. Performance of a fluid-based, thin-layer Papanicolaou smear method in the clinical setting of an independent laboratory and an outpatient screening population in New England. Arch Pathol Lab Med 1999;123:817-21.

6 Weintraub J, Morabia A. Efficacy of a liquid-based thin layer method for cervical cancer screening in a population with a low incidence of cervical cancer. Diagn Cytopathol 2000;22:52-9.

7 Lee KR, Ashfaq R, Birdsong GG, et al. Comparison of conventional Papanicolaou smears and a fluid-based, thin-layer system for cervical cancer screening. Obstet Gynecol 1997;90:278-84.

8 Lin WM, Ashfaq R, Michalopulos EA, et al. Molecular Papanicolaou tests in the twenty-first century: molecular analyses with fluid-based Papanicolaou technology. Am J Obstet Gynecol 2000; 183:39-45.

9 Fiel-Gan MD, Villamil CF, Mandavilli SR, et al. Rapid detection of HSV from cytologic specimens collected into ThinPrep fixative. Acta Cytol 1999;43:1034-8.

10 Inhorn SL, Wand PJ, Wright TC, et al. Chlamydia trachomatis and Pap testing from a single, fluid-based sample. A multicenter study. J Reprod Med 2001;46:237-42.

11 Bianchi A, Moret F, Desrues JM, et al. PreservCyt transport medium used for the ThinPrep Pap test is a suitable medium for detection of Chlamydia trachomatis by the COBAS Amplicor CT/NG test: results of a preliminary study and future implications. J Clin Microbiol 2002;40:1749-54.

12 Mallinson H, Hopwood J, Mutton K. Resolution of the recent performance problem of Abbott LCx Chlamydia trachomatis assay. Issues of repeat testing for confirmation of chlamydial infection. Sex Transm Infect 2002;78:225-6. 